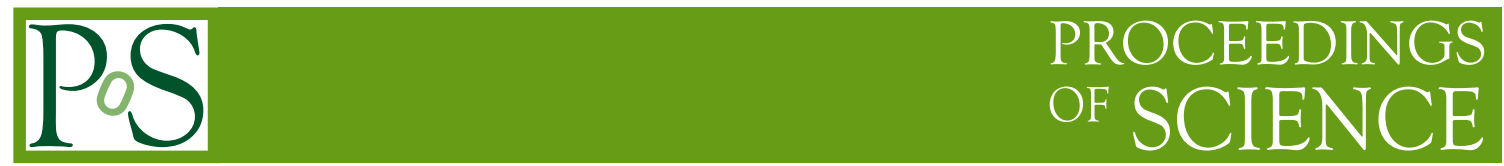

\title{
Helicity amplitudes with off-shell partons
}

\author{
A. van Hameren* \\ The Henryk Niewodniczański Institute of Nuclear Physics \\ Polish Academy of Sciences \\ E-mail: hamerendifj.edu.pl
}

High-energy factorization, or $\mathrm{k}_{\mathrm{T}}$-factorization, requires matrix elements with off-shell initialstate partons. Recently, it has been shown how scattering amplitudes with an arbitrary number of off-shell gluons can be defined in a manifestly gauge invariant way. Here, we show how such amplitudes can be calculated via BCFW recursion.

Loops and Legs in Quantum Field Theory - LL 2014,

27 April - 2 May 2014

Weimar, Germany

${ }^{*}$ Speaker. 


\section{Introduction}

The feasibility of calculations for the prediction of observables for high-energy hadron collider experiments like at the LHC depends on the applicability of factorization prescriptions. These allow for the separation of the process-dependent ingredients, which are calculable perturbatively, from the universal ingredients, which must at least partly be determined from experimental data.

The process dependent ingredients are given by scattering amplitudes. Within collinear factorization, the momenta of the particles and partons associated with an amplitude are on-shell, and the amplitude is defined applying the Lehmann Symanzik Zimmermann reduction prescription on a connected Green function. In case the dynamics of the process under consideration is governed by a gauge theory, like quantum chromo dynamics (QCD), this approach ensures the necessary gauge invariance. For high-energy factorization or $\mathrm{k}_{\mathrm{T}}$-factorization [1-4], however, the initial-state momenta need to be off-shell, and the above approach cannot be applied directly.

There exist a few approaches in literature to define and calculate scattering amplitudes with off-shell partons. Firstly, there is an effective action approach [5,6], which introduces two new fields, the reggeon fields, each of which represents an off-shell gluon with a given longitudinal momentum associated with it. They enter the QCD Lagrangian via terms similar to Wilson lines, ensuring gauge invariance of the eventual scattering amplitudes involving these fields. A similar approach for off-shell initial-state quarks can be found in [7].

The effective action approach is not ideal for the automation of the numerical calculation of helicity amplitudes with increasing number of external particles. It requires the determination of vertices of higher multiplicity, while efficient methods for numerical calculation of helicity amplitude calculation preferably admit at most four-point vertices. In this light, another approach to define amplitudes with off-shell gluons was developed [8], leading to the same amplitudes as with the effective action approach, but more suitable for automated numerical calculation. In this method, the process with the off-shell gluons is embedded in a larger process involving auxiliary quarks. The initial-state off-shell gluons are replaced by on-shell quarks, and on-shell quarks are added to the final state. The on-shellness insures a gauge invariant definition of the amplitude, and the eikonal Feynman rules the auxiliary quarks turn out to follow lead to the same amplitudes as the ones obtained with the effective action approach. Also this method was generalized to deal with off-shell initial-state quarks [9].

Wilson lines always appear in one form or another in mentioned approaches, and this observation was put in a general framework in [10]. There a manifestly gauge invariant and constructive definition of scattering amplitudes with an arbitrary number of off-shell external gluons was presented, by considering matrix elements of Fourier transforms of straight infinite Wilson line operators associated with the off-shell external gluons. In this work, it becomes particularly clear that what allows the gauge invariant definition is that with each with off-shell external momentum $k^{\mu}$, a direction $p^{\mu}$ is associated, which satisfies $p \cdot k=0$, and with each direction, an eikonal line is associated.

Here, we will indicate how tree-level multi-gluon amplitudes with an arbitrary number of them off-shell can be calculated via Britto Cachazo Feng Witten (BCFW) recursion $[11,12]$. This method has proven to lead to very compact expressions with relatively little effort in case of helicity amplitudes with only on-shell partons. We will illustrate how the aforementioned definitions of 
amplitudes with off-shell partons allow for a straightforward generalization of the BCFW recursive relations, allowing again for compact expressions with relatively little effort. A more detailed treatment can be found in [13].

\section{Definitions}

A helicity amplitude for $n$ external gluons is a function of the momenta $k_{1}, k_{2}, \ldots, k_{n}$ and the directions $p_{1}, p_{2}, \ldots, p_{n}$, satisfying the conditions

$$
\begin{aligned}
k_{1}^{\mu}+k_{2}^{\mu}+\cdots+k_{n}^{\mu}=0 & \text { momentum conservation } \\
p_{1}^{2}=p_{2}^{2}=\cdots=p_{n}^{2}=0 & \text { light-likeness } \\
p_{1} \cdot k_{1}=p_{2} \cdot k_{2}=\cdots=p_{n} \cdot k_{n}=0 & \text { eikonal condition }
\end{aligned}
$$

Notice that, in order to deal with time-like momenta, the directions need to be allowed to have complex-valued components. We will consider only color-ordered or dual amplitudes here, which consist of planar graphs following the Feynman rules

$$
\begin{aligned}
& \mu-v=\frac{-\eta^{\mu \nu}}{\mathrm{K}^{2}} \quad-=\frac{1}{2 p \cdot \mathrm{K}} \quad \prod_{\mu}=\sqrt{2} \mathrm{p}^{\mu} \\
& \chi_{1}^{2}=\frac{1}{\sqrt{2}}\left[\left(K_{1}-K_{2}\right)^{\mu_{3}} \eta^{\mu_{1} \mu_{2}}+\left(K_{2}-K_{3}\right)^{\mu_{1}} \eta^{\mu_{2} \mu_{3}}+\left(K_{3}-K_{1}\right)^{\mu_{2}} \eta^{\mu_{3} \mu_{1}}\right] \\
& { }_{1}^{2} X_{4}^{3}=\frac{-1}{2}\left[2 \eta^{\mu_{1} \mu_{3}} \eta^{\mu_{2} \mu_{4}}-\eta^{\mu_{1} \mu_{2}} \eta^{\mu_{3} \mu_{4}}-\eta^{\mu_{1} \mu_{4}} \eta^{\mu_{2} \mu_{3}}\right]
\end{aligned}
$$

The thick lines refer to gluons, and the thin lines refer the eikonal quarks. The symbol $p$ refers to the direction associated with the eikonal quark line and the symbol $\mathrm{K}$ refers to momentum flowing through a propagator or into a vertex. In the graphical representation of an amplitude, the two external eikonal quark lines are bent together to represent a single off-shell gluon. For example for the process $\emptyset \rightarrow g^{*} g^{*} g$ we have

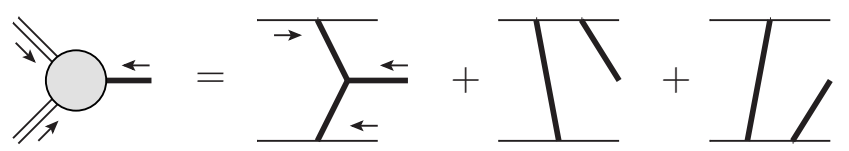

BCFW recursion is based on the fact that tree-level amplitudes are rational functions of the momenta, and on the pole structure of these functions in particular. The theory of complex functions, and Cauchy's theorem in particular, are naturally applied, requiring amplitudes to be defined for complex external momenta. This analytic continuation just has to be performed such that momentum conservation and on-shellness are not broken. With off-shell gluons, the amplitude is still a rational function, now involving also the directions. For the off-shell gluons, the analytic continuation only happens with the momenta, not with the directions. Now, it has to be performed without breaking the eikonal condition (2.3). 
In the original BCFW approach, two adjacent external momenta, say $k_{1}^{\mu}$ and $k_{n}^{\mu}$ are shifted

$$
k_{1} \rightarrow \hat{k}_{1}^{\mu}(z) \equiv k_{1}^{\mu}+z e^{\mu} \quad, \quad k_{n} \rightarrow \hat{k}_{n}^{\mu}(z) \equiv k_{n}^{\mu}-z e^{\mu},
$$

where $z$ is a complex variable, and where the shift vector $e^{\mu}$ is such that $e \cdot e=k_{1} \cdot e=k_{n} \cdot e=0$. So both momentum conservation and on-shellness are guaranteed. In our approach, the same momenta are shifted, but with the shift vector connected to the directions:

$$
e \cdot e=p_{1} \cdot e=p_{n} \cdot e=0,
$$

so that now the eikonal condition is guaranteed to hold. The shift vector can be constructed the same way as in the original BCFW approach, but in terms of the directions (the definition of the spinors can be found for example in [8]):

$$
e^{\mu}=\frac{1}{2}\left\langle p_{1}\left|\gamma^{\mu}\right| p_{n}\right] \quad \text { or } \quad e^{\mu}=\frac{1}{2}\left\langle p_{n}\left|\gamma^{\mu}\right| p_{1}\right] .
$$

For the on-shell case, the gluons should have opposite helicity, and for example the first shift vector applies when gluon 1 has helicity minus. For the off-shell case, either can be used. For mixed cases, the helicity of the on-shell gluon determines which shift vector applies.

For an on-shell gluon, the shift vector is essentially its polarization vector. Let us try to understand its relation to an off-shell gluon. With the help of an auxiliary four-vector $q^{\mu}$ with $q^{2}=0$, the transverse vector of the off-shell momentum $\mathrm{k}^{\mu}$ can be defined by

$$
k_{T}^{\mu}(\mathbf{q})=k^{\mu}-x(\mathbf{q}) p^{\mu} \quad \text { with } \quad x(\mathbf{q}) \equiv \frac{\mathbf{q} \cdot k}{\mathbf{q} \cdot \mathbf{p}} .
$$

With this definition, $k_{T}^{\mu}$ satisfies both the relations $p \cdot k_{T}=0$ and $q \cdot k_{T}=0$. The transverse vector can be constructed explicitly in terms of $p^{\mu}$ and $q^{\mu}$, and we can write

$$
k^{\mu}(q)=x(q) p^{\mu}-\frac{\kappa}{2} \frac{\left\langle p\left|\gamma^{\mu}\right| q\right]}{[p q]}-\frac{\kappa^{*}}{2} \frac{\left\langle q\left|\gamma^{\mu}\right| p\right]}{\langle q p\rangle} \quad \text { with } \quad \kappa=\frac{\langle q|k| p]}{\langle q p\rangle}, \kappa^{*}=\frac{\langle p|k| q]}{[p q]} .
$$

The virtuality of the off-shell momentum can then be written as

$$
\mathrm{k}^{2}=-\mathrm{k}^{*} \text {. }
$$

It turns out that $k$ and $k^{*}$ are both individually independent of $q^{\mu}$. For any light-like $r^{\mu} \neq p^{\mu}$, we have

$$
\kappa=\frac{\langle q|k| p]}{\langle q p\rangle}=\frac{\langle q|k| p]\langle p r\rangle}{\langle q p\rangle\langle p r\rangle}=\frac{\langle q|k p| r\rangle}{\langle q p\rangle\langle p r\rangle}=\frac{\langle q|2 k \cdot p-\not p k| r\rangle}{\langle q p\rangle\langle p r\rangle}=-\frac{\langle q p\rangle[p|k| r\rangle}{\langle q p\rangle\langle p r\rangle}=\frac{\langle r|k| p]}{\langle r p\rangle},
$$

where we used that $k \cdot p=0$. Similarly the independence of $k^{*}$ from $q^{\mu}$ can be demonstrated.

Using the direction $p_{n}^{\mu}$ to define the transverse momentum of gluon 1 and vice versa, we see from (2.10) that the shift (2.6) results in a shift of $\kappa_{1}^{*}, K_{n}$, or $\kappa_{1}, K_{n}^{*}$, depending on the choice (2.8) of shift vector. This is the off-shell equivalent of the shifting spinors in on-shell BCFW. Summarizing, we have

$$
\begin{aligned}
& \hat{\mathrm{k}}_{1}^{\mu}=\mathrm{k}_{1}^{\mu}+\frac{z}{2}\left\langle\mathrm{p}_{1}\left|\gamma^{\mu}\right| \mathrm{p}_{\mathrm{n}}\right] \quad \hat{\mathrm{k}}_{\mathrm{n}}^{\mu}=\mathrm{k}_{\mathrm{n}}^{\mu}-\frac{z}{2}\left\langle\mathrm{p}_{1}\left|\gamma^{\mu}\right| \mathrm{p}_{\mathrm{n}}\right] \\
& 1^{*} \text { off-shell: } \quad \hat{\kappa}_{1}=\kappa_{1}-z\left[p_{1} p_{n}\right] \quad n^{*} \text { off-shell: } \quad \hat{\kappa}_{n}^{*}=\kappa_{n}^{*}+z\left\langle p_{1} p_{n}\right\rangle \\
& \left.\left.\left.1^{-} \text {on-shell: } \mid \hat{p}_{1}\right]=\mid p_{1}\right]+z \mid p_{n}\right] \quad n^{+} \text {on-shell: }\left|\hat{p}_{n}\right\rangle=\left|p_{n}\right\rangle-z\left|p_{1}\right\rangle
\end{aligned}
$$




\section{Recursive relation}

Like in the original BCFW approach, recursive relations for the amplitudes can be derived analyzing the pole structure and the behavior at infinity of the function

$$
\hat{\mathcal{A}}(z) \equiv \mathcal{A}\left(\hat{k}_{1}(z), k_{2}, \ldots, k_{n-1}, \hat{k}_{n}(z)\right),
$$

where $\hat{\mathcal{A}}(0)=\mathcal{A}\left(k_{1}, k_{2}, \ldots, k_{n-1}, k_{n}\right)$ is a color-ordered $n$-gluon amplitude. It is crucial that this function vanishes for $z \rightarrow \infty$. Since eikonal quarks at most add powers of $z$ to the denominator of this function when external momenta are shifted, the reasoning of on-shell BCFW still goes through, up to the issue of the polarization vectors. In the on-shell case, these add a power of $z$ to the denominator of the amplitude, ensuring $\hat{\mathcal{A}}(z \rightarrow \infty)=0$. In order to ensure this in the offshell case, the full propagator denominators of the off-shell external gluons must be included in the amplitudes. Only after an expression of such an amplitude is found, after the recursive procedure, it must be multiplied by a factor proportional to the square root of the virtuality of the off-shell external gluon, in order to get the right on-shell limit if this virtuality is taken to zero [8].

Analyzing the pole structure of the amplitudes in the graphical representation, the recursive relation can straightforwardly be derived to be

$$
2 \bigodot_{n}^{n-1}=\sum_{i=2}^{n-2} \sum_{h=+,-} A_{i, h}+\sum_{i=2}^{n-1} B_{i}+C+D \text {, }
$$

where

$$
A_{i, h}=: \overbrace{\hat{i}}^{i}=\frac{1}{K_{1, i}^{2}}-h:
$$$$
B_{i}=\bigcup_{\hat{i}}^{i-1}-\frac{1}{2 p_{i} \cdot k_{i, n}}-\bigcap_{\hat{n}}^{i}:
$$$$
\mathrm{D}=\frac{1}{\mathrm{~K}_{\mathrm{n}}^{*}}
$$

The hatted numbers label the shifted external gluons. We use the convention that double lines may refer to both off-shell and on-shell external gluons, and that only when we wish to specify that an external gluon is on-shell, we represent it by a thick solid line. For internal momenta, we adopt the notation

$$
\mathrm{K}_{i, j}^{\mu} \equiv k_{i}^{\mu}+k_{i+1}^{\mu}+\cdots+k_{j}^{\mu} .
$$

The terms $A_{i, h}$ correspond to the usual contribution in the BCFW construction for on-shell gluons. The shift variable for the shifted momenta is given by

$$
\hat{\mathrm{K}}_{1, \mathrm{i}}(z)=0 \Rightarrow z=-\frac{\mathrm{K}_{i, n}^{2}}{2 e \cdot \mathrm{K}_{\mathrm{i}, \mathrm{n}}}=-\frac{\mathrm{K}_{i, \mathrm{n}}^{2}}{\left\langle\mathrm{p}_{1}\left|\mathrm{~K}_{\mathrm{i}, \mathrm{n}}\right| \mathrm{p}_{\mathrm{n}}\right]} .
$$

The two blobs represent well-defined amplitudes with an on-shell gluon referred to by the thick line, and the sum is over the possible helicities of these on-shell gluons. 
The two blobs in terms of type $B_{i}$ correspond to amplitudes with an off-shell gluon with direction $p_{i}^{\mu}$, associated with the cut eikonal line. The shift variable is now given by

$$
p_{i} \cdot \hat{K}_{i, n}(z)=0 \Rightarrow z=-\frac{2 p_{i} \cdot K_{i, n}}{2 p_{i} \cdot e}=-\frac{2 p_{i} \cdot K_{i, n}}{\left\langle p_{1} p_{i}\right\rangle\left[p_{i} p_{n}\right]}
$$

It has to be noted that the momentum of an off-shell gluon can be freely distributed over the two external eikonal lines associated with the gluon, as long as the inner product of the direction with each of the momentum fractions vanishes. Exactly this ensures that the two blobs are well-defined off-shell amplitudes. The term $B_{i}$ is is absent if $i$ labels an on-shell external gluon.

As mentioned before, the amplitudes under consideration must include the propagator denominator of the external off-shell gluons, giving rise to the terms $C$ and D. These only appear if gluon 1 and $n$ are off-shell respectively. They become on-shell with shifted momenta

$$
\hat{k}_{1}^{\mu} \equiv \hat{k}_{1}^{\mu}\left(z=\kappa_{1} /[1 n]\right)=x_{1}\left(p_{n}\right) p_{1}^{\mu}-\frac{\kappa_{1}^{*}}{2} \frac{\left\langle n\left|\gamma^{\mu}\right| 1\right]}{\langle n 1\rangle}
$$

for the term $\mathrm{C}$, and

$$
\hat{k}_{n}^{\mu} \equiv \hat{k}_{n}^{\mu}\left(z=-\kappa_{n}^{*} /\langle n 1\rangle\right)=x_{n}\left(p_{1}\right) p_{n}^{\mu}-\frac{\kappa_{n}}{2} \frac{\left\langle n\left|\gamma^{\mu}\right| 1\right]}{[n 1]}
$$

for the term D.

\section{Results}

A question that immediately arises is how an amplitude with an off-shell gluon is related to the amplitudes for which this gluon is on-shell. The plural is appropriate here, because the onshell gluon may come in two helicities. It turns out that the helicity amplitudes are embedded in the off-shell amplitudes as separate terms. This coherent sum of terms becomes an incoherent sum of squared amplitudes in the on-shell limit of the squared amplitude via the residual angular integration that has to be performed for the transverse momentum when its magnitude is taken to zero. This integration eliminates the interference terms of different helicities. One of the helicity amplitudes for the process $\emptyset \rightarrow g^{*} g g g^{*}$, for example, is found in [13] to be

$$
\begin{aligned}
\mathcal{A}\left(1^{*}, 2^{+}, 3^{-}, 4^{*}\right) & =\frac{1}{\kappa_{1}^{*} \kappa_{4}} \frac{-\left\langle 1\left|p_{3}+k_{4}\right| 4\right]^{4}}{\left\langle 2\left|k_{1}\right| 4\right]\left\langle 1\left|k_{4}\right| 3\right]\langle 12\rangle[43]\left(p_{3}+k_{4}\right)^{2}} \\
& +\frac{1}{k_{1}} \frac{\langle 34\rangle^{3}[14]^{3}}{\left\langle 4\left|k_{4}+k_{1}\right| 1\right]\left\langle 2\left|k_{1}\right| 4\right]\left\langle 4\left|k_{1}\right| 4\right]\langle 23\rangle}+\frac{1}{\kappa_{4}^{*}} \frac{[21]^{3}\langle 14\rangle^{3}}{\left\langle 4\left|k_{4}+k_{1}\right| 1\right]\left\langle 1\left|k_{4}\right| 3\right]\left\langle 1\left|k_{4}\right| 1\right][32]} .
\end{aligned}
$$

Here, we use the short-hand notation $|i\rangle \equiv\left|p_{i}\right\rangle$ etc.. The first term multiplied with $\kappa_{1}^{*} \kappa_{4}$ gives $\mathcal{A}\left(1^{-}, 2^{+}, 3^{-}, 4^{+}\right)$in the on-shell limit, while the second and the third, multiplied with $\mathrm{K}_{1} \mathrm{~K}_{4}^{*}$, combine to give $\mathcal{A}\left(1^{+}, 2^{+}, 3^{-}, 4^{-}\right)$. Realize that $\mathcal{A}\left(1^{+}, 2^{+}, 3^{-}, 4^{+}\right)=\mathcal{A}\left(1^{-}, 2^{+}, 3^{-}, 4^{-}\right)=0$.

A well-known phenomenon in multi-gluon helicity amplitudes is that they vanish for helicity configurations in which all, or all but one gluon have the same helicity, and come in particularly simple expressions for the case in which all but two gluons have the same helicity, the so-called 
maximum-helicity-violating (MHV) configurations. It turns out that these are exactly the expressions for helicity amplitudes with two off-shell gluons, for which all on-shell gluons have the same helicity:

$$
\begin{aligned}
\mathcal{A}\left(1^{*}, i^{*},(\text { the rest })^{+}\right) & =\frac{1}{\kappa_{1}^{*} \kappa_{i}^{*}} \frac{\left\langle p_{1} p_{i}\right\rangle^{4}}{\left\langle p_{1} p_{2}\right\rangle\left\langle p_{2} p_{3}\right\rangle \cdots\left\langle p_{n-2} p_{n-1}\right\rangle\left\langle p_{n-1} p_{n}\right\rangle\left\langle p_{n} p_{1}\right\rangle} \\
\mathcal{A}\left(1^{*}, i^{*},(\text { the rest })^{-}\right) & =\frac{1}{\kappa_{1} \kappa_{i}} \frac{\left[p_{i} p_{1}\right]^{4}}{\left[p_{1} p_{n}\right]\left[p_{n} p_{n-1}\right]\left[p_{n-1} p_{n-2}\right] \cdots\left[p_{3} p_{2}\right]\left[p_{2} p_{1}\right]} .
\end{aligned}
$$

\section{Conclusions}

Calculations of scattering processes performed within high-energy factorization or $k_{T}$-factorization require matrix elements with off-shell initial state partons. Recent developments have led to a general manifestly gauge-invariant definition of scattering amplitudes with an arbitrary number of off-shell gluons. BCFW recursion has been generalized for application to such amplitudes, and using this approach, compact expressions have been derived for them.

\section{Acknowledgement}

This work was partially supported by NCBiR grant LIDER/02/35/L-2/10/NCBiR/2011.

\section{References}

[1] L. Gribov, E. Levin, and M. Ryskin, Phys.Rept. 100 (1983) 1-150.

[2] S. Catani, M. Ciafaloni, and F. Hautmann, Nucl.Phys. B366 (1991) 135-188.

[3] J. C. Collins and R. K. Ellis, Nucl.Phys. B360 (1991) 3-30.

[4] S. Catani and F. Hautmann, Nucl.Phys. B427 (1994) 475-524, [hep-ph / 9405388 ].

[5] L. Lipatov, Nucl.Phys. B452 (1995) 369-400, [hep-ph/9502308].

[6] E. Antonov, L. Lipatov, E. Kuraev, and I. Cherednikov, Nucl.Phys. B721 (2005) 111-135, [hep-ph/0411185].

[7] L. Lipatov and M. Vyazovsky, Nucl.Phys. B597 (2001) 399-409, [hep-ph / 0009340 ].

[8] A. van Hameren, P. Kotko, and K. Kutak, JHEP 1301 (2013) 078, [1211.0961].

[9] A. van Hameren, K. Kutak, and T. Salwa, Phys.Lett. B727 (2013) 226-233, [1308 . 2861].

[10] P. Kotko, 1403.4824.

[11] R. Britto, F. Cachazo, and B. Feng, Nucl.Phys. B715 (2005) 499-522, [hep-th/ 0412308 ].

[12] R. Britto, F. Cachazo, B. Feng, and E. Witten, Phys.Rev.Lett. 94 (2005) 181602, [hep-th/0501052].

[13] A. van Hameren, 1404.7818. 